\title{
The Realization of Math Score Query on the Wireless Internet
}

\author{
Guang Yang \\ Department of Basic Courses Teaching The Armed Police Academy Langfang City \\ Hebei Province, China \\ E-mail: yg_sun1981@sina.com
}

\begin{abstract}
Wireless internet is gaining wide spread usage in teaching affairs, especially in score query system development. This paper explores how simple Math score query system can be developed for mobile devices and introduces general framework for designing such score query applications. Using the framework, based on Microsoft database software SQL Server 2000, T-SQL clause is employed to program one stored procedure for Math score query. The framework utilizes mobile phones simulator software, Nokia WAP Toolkit, which interacts with a server-side ASP (Active Server Pages) in conjunction with the associated SQL Server database. The design and implementation of the management information system are discussed in the paper, and test results are presented.
\end{abstract}

Keywords: Score Query System; T-SQL; WAP Toolkit; ASP; SQL Server; Framework

\section{I .INTRODUCTION}

Management information systems[1] are becoming more and more popular in the information age[1] and the wireless Internet[2] is a new revolution upon us, one that will affect the world on a scale similar to that of the wired Internet. This paper demonstrates the general framework for designing online score query management information system on the wireless internet. In the teaching affairs system [3], test score query is very popular for the students; for the purpose of demonstration of the framework, Math score query application on the wireless network [4] is developed. This framework utilizes Microsoft Active Server Pages (ASP)[5][6] in conjunction with the associated SQL Server[7] database to create a truly dynamic application using WML (Wireless Markup Language)[8] to develop application on the mobile phones.

\section{II .DATABASE DESIGN AND IMPLEMENTATION}

A. Design the Database

SQL Server 2000[7] is used as the database to provide the solution for Math score query. At first, we design one database: MathTestScore and then we design the related tables in this database to save the students' basic information, their learning semester information and their Math test score information, they are stud_basic_info, semester_info and testscore_info.The design of these three tables are shown in TABLE1, TABLE2 and TABLE3.

TABLE I. DESIGN OF THE DATA TABLE: STUD_BASIC_INFO

\begin{tabular}{|c|l|l|}
\hline StudID & Varchar(30) & Student Number \\
\hline StudName & Varchar(30) & Name of the Student \\
\hline Gender & bit & 1 stands for male and 0 stands for female \\
\hline Birthdate & Datetime & Birthdate of the Student \\
\hline
\end{tabular}

TABLE II. DESIGN OF THE DATA TABLE: SEMESTER_INFO

\begin{tabular}{|c|l|l|}
\hline ColumnName & $\begin{array}{l}\text { Data } \\
\text { Type }\end{array}$ & Remarks \\
\hline SemesterID & int & $\begin{array}{l}\text { Identity of the semester, Automatic } \\
\text { Increase by } 1\end{array}$ \\
\hline SemesterYear & int & Semester Year \\
\hline Semester & tinyint & First Semester or the Second Semester \\
\hline
\end{tabular}

TABLE III. DESIGN OF THE DATA TABLE : TESTSCORE_INFO

\begin{tabular}{|c|l|l|}
\hline ColumnName & Data Type & Remarks \\
\hline ScoreID & int & Automatic Increase by 1 \\
\hline SemesterID & int & $\begin{array}{l}\text { Reference the StudID column of table } \\
\text { Stud_Basic_Info }\end{array}$ \\
\hline CourseName & Varchar(50) & $\begin{array}{l}\text { Reference the SemesterID column of } \\
\text { table Semester_Info }\end{array}$ \\
\hline TestType & Varchar(10) & Test Type,Quiz or Final \\
\hline TestObjective & Varchar(20) & Theory or Application \\
\hline TestScore & Varchar(10) & A,A+,95 or other types of score \\
\hline TestDate & Datetime & Test Date \\
\hline
\end{tabular}

B. Program the Stored Procedure for the Query

In respect to the three tables: Stud_Basic_Info, Term_Info and Testscore_Info, we design one view[7] VW_TestScore for the convenience of query, and the source codes are shown as follows.

Create View VW_ TestScore As

SELECT stud_basic_info.StudID,stud_basic_info.StudName, stud_basic_info.Gender,stud_basic_info.Birthdate,semester_info.semestery ear,semester_info.semester AS Semester, testscore_info.coursename, testscore_info.testtype, 
testscore_info.testobjective, testscore_info.testscore,

testscore_info.testdate FROM stud_basic_info

INNER JOIN testscore_info ON

stud_basic_info.StudID =testscore_info.studid INNER JOIN semester_info ON testscore_info.semesterid =semester_info.semesterid

In order to simplify the query clause, which is called by ASP web files, we use the processing tool-----Query Analyzer[1] on the platform of SQL Server 2000 to program one stored procedure[10][11] SP_TestscoreQuery with T-SQL[1] and the source codes are shown as follows. CREATE PROC SP_ TestscoreQuery

@StudID varchar(30),

@StudName varchar(50)

AS

BEGIN

Select * from VW_ TestScore where studid=@StudID and studname=@StudName

END

\section{Program the Stored Procedure for the Query}

We assume there are 3 students and they have finished 2 term years Math learning, i.e. 4 semesters. The sample data are shown in TABLE 4,5,6.

TABLE4. SAMPLE DATA OF TABL: STUD_BASIC_INFO

\begin{tabular}{|c|l|l|l|}
\hline \multicolumn{1}{|c|}{ StudID } & StudName & Gender & Birthdate \\
\hline 20095082107 & LIU Tao & 1 & $1991-4-30$ \\
\hline 20084083219 & WANG Zhi & 1 & $1989-7-13$ \\
\hline 20103092238 & ZHAO Yong & 0 & $1992-10-25$ \\
\hline
\end{tabular}

TABLE5. SAMPLE DATA OF TABLE : TERM_INFO

\begin{tabular}{|c|l|l|}
\hline SemesterID & SemesterYear & Semester \\
\hline 1 & 2009 & 1 \\
\hline 2 & 2009 & 2 \\
\hline 3 & 2010 & 1 \\
\hline 4 & 2010 & 2 \\
\hline
\end{tabular}

TABLE6. SAMPLE DATA OF TABLE : TESTSCORE_INFO

\begin{tabular}{|c|c|c|c|c|c|c|c|}
\hline $\begin{array}{l}\text { Score } \\
\text { ID }\end{array}$ & SudIID & $\begin{array}{l}\text { Semeste } \\
\mathrm{ID}\end{array}$ & $\begin{array}{l}\text { CounseN } \\
\text { ame }\end{array}$ & $\begin{array}{l}\text { TestT } \\
\text { ype }\end{array}$ & $\begin{array}{l}\text { TestObje } \\
\text { dive }\end{array}$ & $\begin{array}{l}\text { TestSc } \\
\text { ore }\end{array}$ & $\begin{array}{l}\text { Test } \\
\text { Date }\end{array}$ \\
\hline 1 & $\begin{array}{l}20095082 \\
107\end{array}$ & 2 & $\begin{array}{l}\text { Linear } \\
\text { Algebar }\end{array}$ & $\begin{array}{l}\text { Qui } \\
\text { z }\end{array}$ & Matrix & $\mathrm{B}+$ & $\begin{array}{l}2010-4 \\
-21\end{array}$ \\
\hline 2 & $\begin{array}{l}20084083 \\
219\end{array}$ & 1 & $\begin{array}{l}\text { Probabilit } \\
\text { y }\end{array}$ & $\begin{array}{l}\text { Fina } \\
l\end{array}$ & $\begin{array}{l}\text { Statisti } \\
\text { Cs }\end{array}$ & 79 & $\begin{array}{l}2010-1 \\
-9\end{array}$ \\
\hline 3 & $\begin{array}{l}20084083 \\
219\end{array}$ & 2 & $\begin{array}{l}\text { Linear } \\
\text { Algebar }\end{array}$ & $\begin{array}{l}\text { Qui } \\
\text { z }\end{array}$ & Matrix & A & $\begin{array}{l}2010-2 \\
-29\end{array}$ \\
\hline 4 & $\begin{array}{l}20095082 \\
107\end{array}$ & 4 & $\begin{array}{l}\text { Caculu } \\
\text { s }\end{array}$ & Quiz & $\begin{array}{l}\text { Definitive } \\
\text { Caculus }\end{array}$ & 88 & $\begin{array}{l}2011-2 \\
-28\end{array}$ \\
\hline
\end{tabular}

The above sample data are inserted into the database and test the stored procedure on Query Analyzer[1]. The testing result is shown in Figure 1.

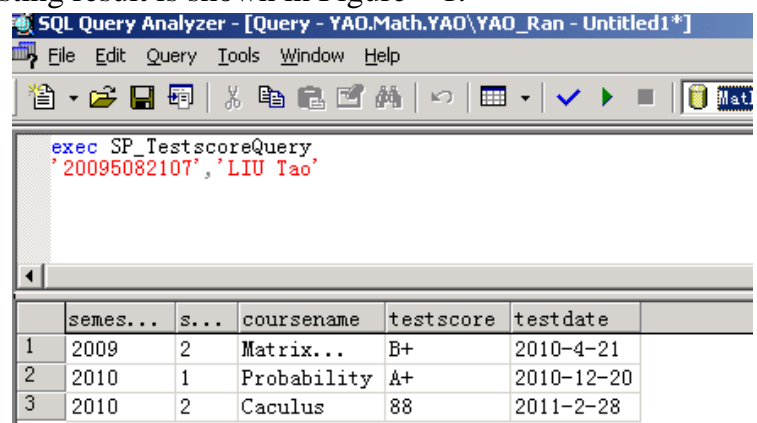

Figure 1 . Test of the stored procedure

\section{SOFTWARE ARCHITECHTURE FOR SCORE QUERY APPLICATION}

Dynamic applications[9] are applications that build content "on the fly" in response to request made. They can reflect the latest traffic information from the database. Microsoft Active Server Pages (ASP)[6] in conjunction with the associated SQL Server[7] database to create a truly dynamic Math test score query application. The conceptual software architecture is shown in Figure 2 and the architecture contains the following components:

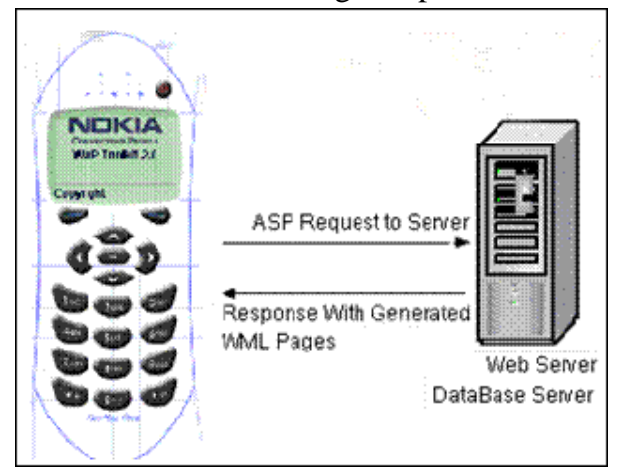

Figure 2. Architecture of the dynamic application

\section{A.WEB Server}

IIS (Internet Information Service)[12] is configured to create one virtual directory(Figure 3) containing one file: query.asp, which connects the database server, gets the query results from the database server and responds with generated WML pages. 
. Internet 'Information Service

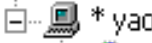
Default Website
+ application
+ MathQuery
$+\square$ AJAXEnabledWebsite 1
+ calendar
calendar 3
+ CrystalReportsWebsite 1
$+\square$ CSSMASTERPAGE
$+\square$ dataupdate

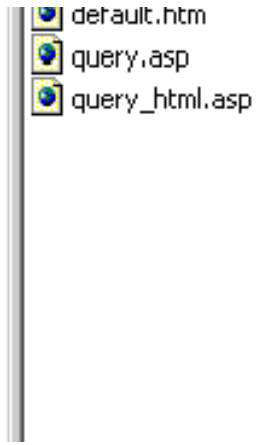

Figure 3. Configuration of IIS to create virtual directory

B. DataBase Server(Shown in Part II)

C. Application Design

The client software was developed using Nokia Wap Toolkit[2] 2.0. This kit enables developers to create applications on the mobile phone based on the WAP (Wireless Application Protocol)[2]. Wireless Markup Language (WML) is used to implement the Wireless Application Protocol (WAP) specification and creates pages that can be displayed in a WAP browser. WMLScript [8], a client-side scripting language, is used for developing tasks such as user input validation, generation of error message and other Dialog boxes etc. on the mobile phone. The Toolkit contains tools for editing both WML and WMLScript, and it can display the resulting code on a mobile service simulator[13] (Figure 4). Figure 5 and Figure 6 illustrate the desired outcome of the score query application.

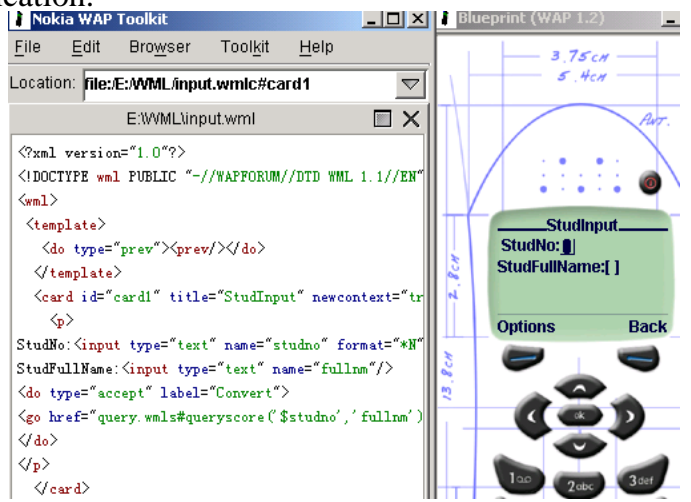

Figure 4. Nokia wap toolkit for editing wml or wmlscript and displaying the resulting code on a mobile service simulator

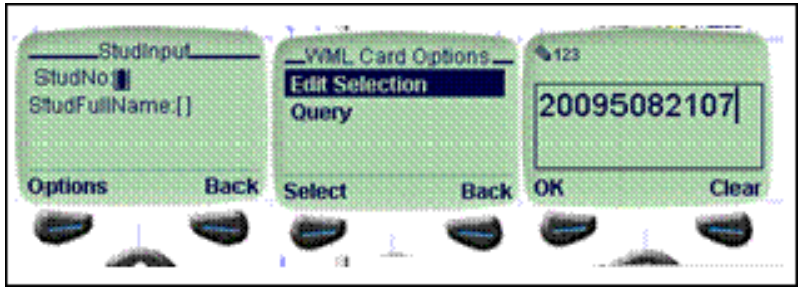

Figure 5. Keying the student number and fullname to query

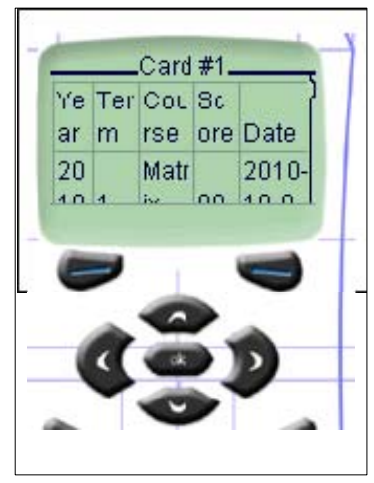

Figure 6. Viewing the Result of the Math Test Score Query

The application consists of the following source files:

1) input.wml: this file is stored on the client mobile phone and is responsible for displaying the user interface and calls the query function defined in query.wmls script to display the Math score query result.

2) query.wmls: this file is stored on the client mobile phone and is invoked by input.wml when the client wants to query the Math score of one student. It sends ASP [6][9] request to the query.asp file stored in Web server.

3) query.asp: this file is stored in the Web server[12] and is automatically invoked when query.wmls script is executed in the client mobile phone. It connects to the database which is stored in Database server, gets the query results from the database server and responds with generated WML pages[8].

\section{Testing Of The Programs}

In order to test programs, Nokia Wap Toolkit[2] is installed to the Web Server whose system software is Window 2000 Server[12]. If we want to query the Math score of this student, whose full name and student number are 20095082107 and LIU Tao. Figure 7, Figure 8, Figure 9 and Figure 10 show the resulting screens on the WAP Toolkit.

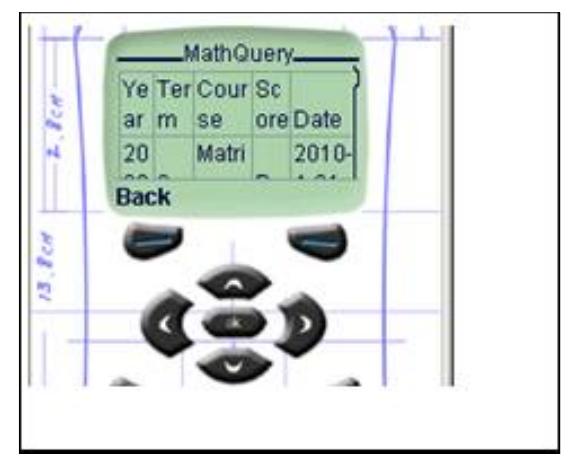

Figure 7. Results for math score query of liu tao 


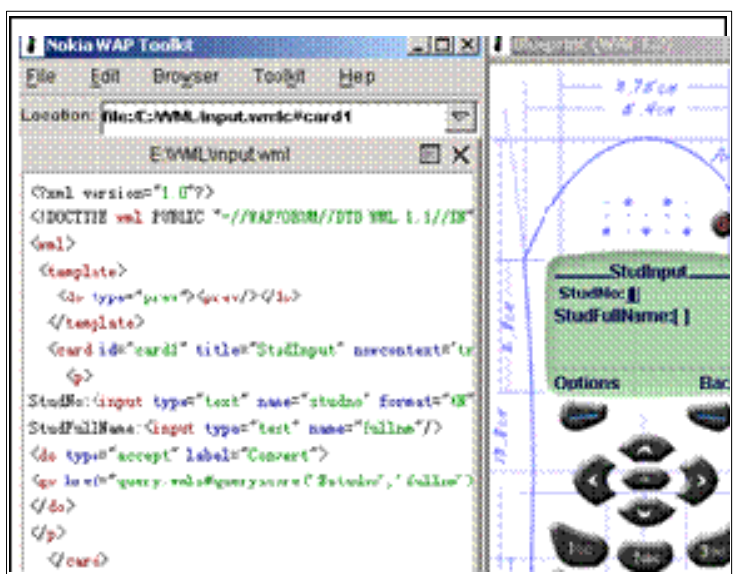

Figure 8. Results for math score query of liu tao

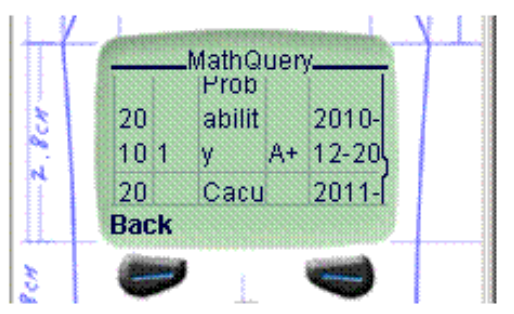

Figure 9. Results for math score query of liu tao

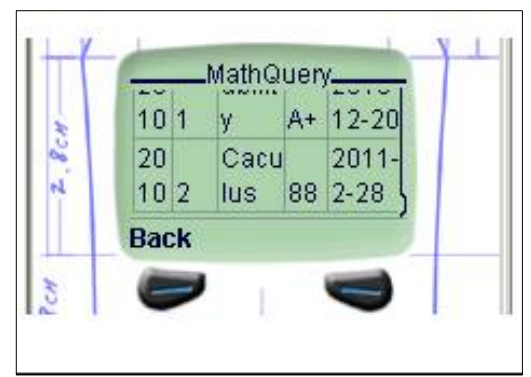

Figure 10. Results for math score query of liu tao (continued)

\section{CONCLUSION}

With the help of the T-SQL clause, the stored procedure of Math test score query is created and tested successfully on the platform of the SQL Server 2000 database; With the application of WAP Toolkit, the WML and WMLScript web files are designed to send the users' request to the Web server, the corresponding dynamic ASP programs[14] are developed to process the request and acquire the score query result from the database server and finally the Math test score query result is shown on the mobile phones with the dynamically generated wml files and the testing result is satisfactory.
This conceptual design framework can easily be used for any learning and teaching score query applications, such as English score query, entrance exam query and employee appraisal query applications. As more and more test score query applications are conducted using mobile phones, this design framework is flexible enough to be used on the wireless internet.

\section{REFERENCES}

[1] Effy Oz , Management Information System Six Editon, Course Technology, January 2008, pp.208-254

[2] Ryan Fife, Wei Meng Lee , Webmaster's Guide to the Wireless Internet, Syngress, December,2001, pp. 114-160.

[3] Yan Jintong Theroy and Practice of Teaching Admistration(in Chinese) Guangxi Normal University Press, July 2007 pp. 33-89

[4] Huw Evans Paul Ashworth, Getting Started With WAP and WML , San Francisco SYBEX,2001 , pp. 170-282

[5] QU Xilong, LI Zhenggeng, Collections of Instances for Dynamic Website Application with ASP+ACCESS(in Chinese), China Machine Press, July 2006 pp12,27,119-134

[6] Scott Mitchell , Designing Active Server Pages , O'Reilly, September 2000 ,pp 182-203

[7] Tobias Thernstrom, Ann Weber, Mike Hotek, MCTS Self-Paced Training Kit (Exam 70-433): Microsoft ${ }^{\circledR}$ SQL Server ${ }^{\circledR}$ 2008-Database Development, Microsoft Press, February 2009, pp:306-453

[8] Martin Frost ,Learning WML \& WMLScript , O’REILLY , October 2000 , pp.54-134.

[9] YAO Zhiqiang,Abhijit Sen,XIA Han "Dynamic Financial Application Framework For The Mobile Phones” Sept 17-19, 20102010 2nd IEEE International Conference on Information and Financial Engineering ICIFE 2010,ChongQing China

[10] CAO Yaohui, "Application with SQL Server Procedure in System Development”, Modern Electronics Technique, 2005 28(20)

[11] Mourad Ouzzani,Athman Bouguettaya, "Query Processing and Optimization on the Web”, Distributed and Parallel Databases, 2004,15, 187-218.

[12] LIU Lian, YANG Guokai , Windows 2000/XP NetWork Construction Technology Application (in Chinese), Beijing Hope Electronics Press , Dec. 2002 241-276

[13] YAO Zhiqiang,LI Chiyuan,XU Ke,XIA Han “The Application of Online Traffic System On The Wireless Internet” ， Dec 28,2010 2010 2nd International Conference on Information and Multimedia Technology HonhKong

[14] Lin He, Yan Chen, Rui Ding, Si Zhang , "The Informationalization of Small and Medium-size Business Based on the ASP Platform” , 2008 - The Seventh Wuhan International E-Business Conference

\section{Appendix. Source codes for Math Test Score Query}

\section{File: input.wml}

$<$ wml $>\quad<$ template $>\quad<$ do type="prev" $><$ prev $/></$ do $>$

$<$ template $>\quad<$ card id="card1" title="StudInput" newcontext="true" $>$ $<$ p $>$ StudNo:<input type="text" name="studno" format="*N" />

StudFullName: $<$ input type="text" name="fullnm"/>

$<$ do type="accept" label="Convert" $>$

$<$ go href="query.wmls\#queryscore('\$studno','fullnm')" />

$</$ do $><$ /p $>\quad<$ card $>\quad<$ /wml $>$

File: query.wmls

extern function queryscore(studno,fullnm)

\{var

str="http://localhost/MathQuery/query.asp?studno="+studno+"\&fullnm=" + fullnm;

WMLBrowser.go(str);\} 


\section{File: query.asp}

$<\%$ response.ContentType="text/vnd.wap.wml"\%>

$<$ ?xml version="1.0"? $><$ wml $><$ template $>$ label="Back" $>\quad<$ go href="input.wml\#card1" $></$ go $>$

$<$ do type="accept" $</$ do $>$ newcontext="true" > Conn=Server.CreateObject("Adodb.Connection")

Conn.Open set rs2=createobject("ADODB.RecordSet") str="exec SP_TestscoreQuery "'\&request("studno")\&"',"'\&request("fullnm")\&"'"

rs2.open str, Conn \%>

$<$ table align="left" columns="5" title="MathScore" $>$

$<$ tr $><$ td $>$ Year $</$ td $><$ td $>$ Term $</$ td $><$ td $>$ Course $</$ td $><$ td $>$ Score $</$ td $><$ td $>$ D ate $</$ td $></$ tr $><\%$ do while not rs2.eof\% $>$

$<$ tr $><$ td $><\%=$ rs2("semesteryear")\% $></$ td $><$ td $><\%=$ rs2("semester")\% $></$ t $\mathrm{d}><\mathrm{td}><\%=$ rs2("coursename")\% $></$ td $><$ td $><\%=$ rs2("testscore")\% $></$ td $>$ $<$ td $><\%=$ rs2("testdate")\% $></$ td $></$ tr $><\%$ rs2.movenext

Loop rs2=nothing\% $><$ table $></$ p $>\quad</$ card $></$ wml $>$ 